\title{
Uptake, yield of neoplasia, and adverse effects of flexible sigmoidoscopy screening
}

\author{
W S Atkin, A Hart, R Edwards, P McIntyre, R Aubrey, J Wardle, S Sutton, J Cuzick, \\ J M A Northover
}

ICRF Colorectal

Cancer Unit, St Mark's Hospital, Harrow W S Atkin

J M A Northover

ICRF Department of

Mathematics,

Statistics, and

Epidemiology, London

R Edwards

J Cuzick

ICRF Health

Behaviour Unit,

Department of

Epidemiology and

Public Health,

University College,

London

J Wardle

S Sutton

Queen Elizabeth II Hospital, Welwyn Garden City

P McIntyre

R Aubrey

Leicester General Hospital, Leicester, UK A Hart

Correspondence to: Dr W S Atkin, ICRF Colorectal Cancer Unit, St Mark's Hospital, Northwick Park, Watford Road, Harrow, Middlesex HA1 3UJ, UK.

Accepted for publication 26 November 1997

\begin{abstract}
Background-A multicentre randomised controlled trial to evaluate screening by "once only" flexible sigmoidoscopy (FS) for prevention of bowel cancer is in progress.

Aims-To pilot the trial protocol examining rates of attendance, yield of neoplasia, and adverse effects.

Subjects-A total of 3540 subjects aged 55-64 years in Welwyn Garden City (WGC) and 19706 in Leicester (LE). Methods-Subjects responding positively to an "interest in screening" questionnaire were randomised to invitation for screening or control arms. Small polyps were removed during screening. Colonoscopy was undertaken for high risk polyps (more than two adenomas, size at least 1 cm, villous histology, severe dysplasia, or malignancy). The remainder were discharged.

Results-In WGC and LE respectively, $59 \%$ and $61 \%$ indicated an interest in screening, of which $74 \%$ and $75 \%$ attended. Adenomas were detected in $10 \%$ and $9 \%$, respectively, and cancers in 7 per 1000 (in both centres), $55 \%$ at Dukes's stage $A$. The colonoscopy referral rate was $6 \%$ in both centres. Mild, short lived bleeding occurred in $3 \%$. One person died following surgery.

Conclusions-Compliance rates, yield of adenomas, and referral rate for colonoscopy were as expected, but cancer detection rates were higher. Adverse effects following sigmoidoscopy or colonoscopy were mild and transient, but there was one postoperative death. A randomised trial is necessary to evaluate fully the risks and benefits of this intervention.

(Gut 1998;42:560-565)
\end{abstract}

Keywords: screening; colorectal cancer; adenomas; sigmoidoscopy; endoscopy; randomised trial

Colorectal cancer remains the second most common cause of death from malignancy in the Western world, with no likelihood of major improvements in mortality through the treatment of symptomatic disease in the near future. Population screening using faecal occult blood tests offers the prospect of a $15 \%$ decrease in mortality ${ }^{12}$; this is mainly due to diagnosis of cancer at an early pathological stage. Flexible sigmoidoscopy (FS), perhaps performed only once around the age of 60 years, has been put forward as a potential method of cancer prevention through identification and removal of premalignant adenomas. ${ }^{3}$ A multicentre randomised controlled trial to test this hypothesis is now in progress in the UK.

In preparation for this trial, issues relating to population compliance, pathological yield, and the adverse effects of the screening process required detailed assessment. An initial small study in Welwyn Garden City included people registered with two general practices; this was followed by a larger study in Leicester, the first full sized centre in the multicentre trial, aimed at allowing various assumptions regarding the above mentioned issues to be assessed in the context of the main trial protocol.

\section{Methods}

STUDY POPULATIONS

The first study in Welwyn Garden City (WGC) included two general practices with 3540 patients in the eligible age range (55-64 years). The second study in Leicester (LE) was more heterogeneous socioeconomically and included 19706 eligible patients from a mixture of general practices (20 in total), varying in size, distance from the hospital, and locale (inner city, suburban, market town, rural).

\section{RECRUITMENT}

Approval was obtained from the local ethics committees. Participating general practitioners were asked to exclude patients with colorectal cancer, inflammatory bowel disease, colorectal endoscopy within the previous two years, or severe illness.

In order to increase compliance rates, a two stage recruitment procedure has been adopted, whereby subjects are entered into the trial if they respond positively to the question, "If you were invited to have the bowel cancer screening test, would you take up the offer?" (fig 1). An "interest in screening" questionnaire was mailed, with an accompanying letter on the patients' practice headed notepaper signed by the senior partner, to all patients in the age range. Brief information about bowel cancer and the screening test was included. A reminder was sent after two weeks to nonresponders. Those returning a completed questionnaire in the prepaid envelope provided and expressing an interest in having the test were randomised to the control or intervention groups in the ratio $2: 1$. The unit of randomisation was the household. An important objective of these studies was to examine uptake rates for each stage of the recruitment procedure. 
Two stage recruitment procedure

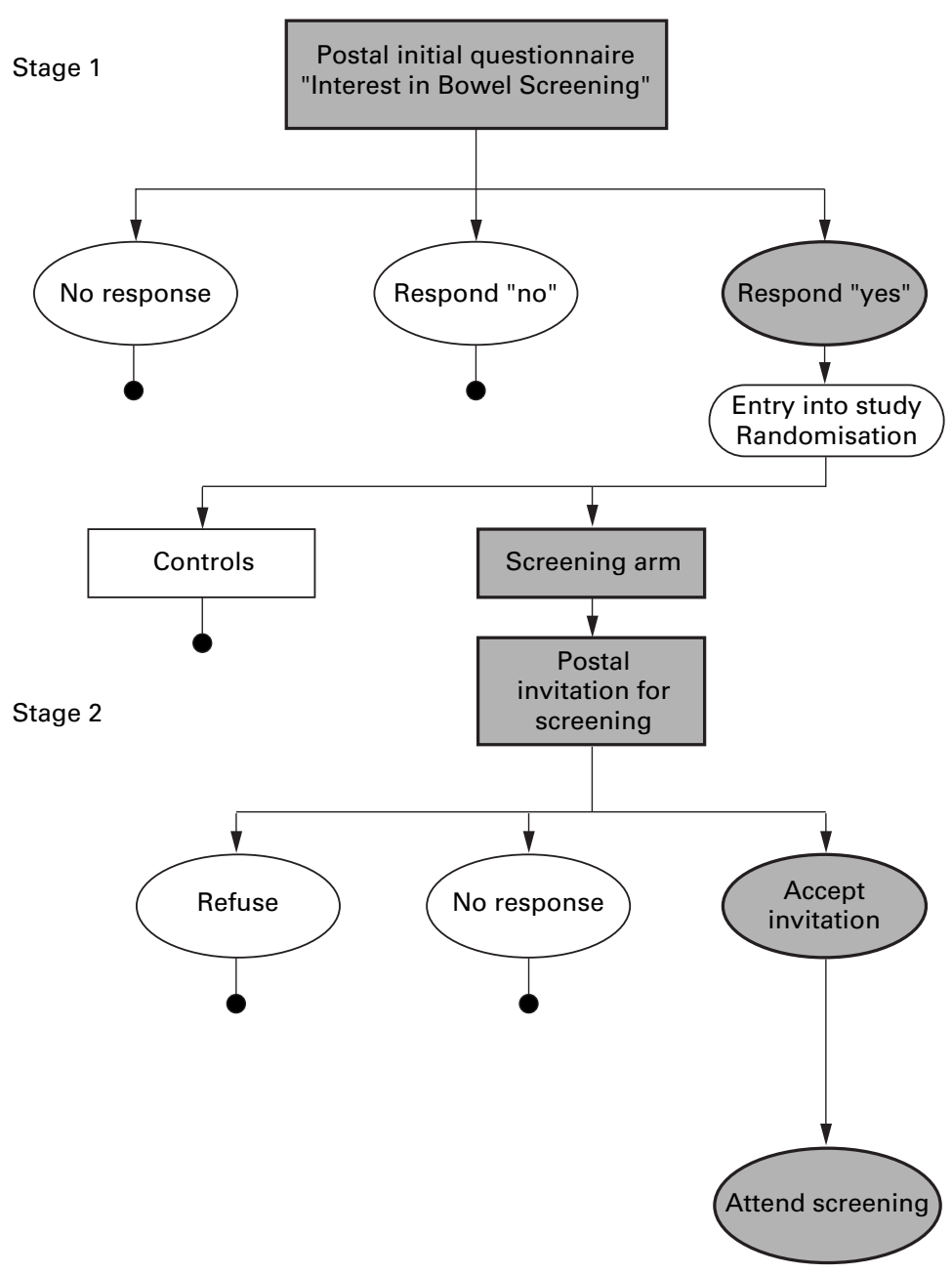

- No further contact, subject followed passively through NHS Central Register of the Office of Populations Censuses and Surveys

Figure 1 Two stage screening procedure.

INVITATION FOR SCREENING

A patient management system was developed (RE) to facilitate the scheduling of appointments and communicating the results of screening. In both centres, screening was performed in the endoscopy unit by experienced specialist gastroenterologists. Invitations with a specified appointment were sent five weeks in advance to those randomised to the intervention arm. Subjects were asked to telephone to confirm, change, or cancel their appointment. To ensure that all available slots in each session were filled, a $50 \%$ overbooking system was used. Initially, in WGC, an attempt was made to telephone the non-responders to encourage them to attend. This proved to be unproductive. Subsequently, a written reminder was sent to non-responders after two weeks. When the original appointment was still available, this was offered again. When it was not available, because of the overbooking system, they were offered an appointment six weeks ahead. Those who had confirmed their appointment were mailed their bowel prepara- tion (either a single sachet of Picolax or a single phosphate enema: Fletchers' Enema, Pharmax Ltd). The efficacy and acceptability of the two forms of bowel preparation offered will be described in a separate paper (in preparation).

\section{SCREENING PROCEDURE}

The flexible sigmoidoscopes (Olympus model CF-2005) were connected to a VDU monitor. The endoscopist advanced the scope as far as could be achieved without producing pain or stress (normally to just beyond the sigmoid colon-descending colon junction). No medication was offered and the examination was not expected to take longer than five minutes. After the examination, participants were given their results by the endoscopist. They were advised to telephone the endoscopy unit if they had any worries in the next few days and to visit their general practitioner if they developed symptoms in the future.

\section{MANAGEMENT OF POLYPS AND SUBSEQUENT INVESTIGATION}

Polyps less than $1 \mathrm{~cm}$ in size were removed during FS screening. Polyps of diameter $1 \mathrm{~cm}$ or larger were removed subsequently at colonoscopy. Following histological examination of small polyps, those with three or more adenomas, tubulovillous or villous histology, or severe dysplasia were sent a letter which advised them of their results and included a colonoscopy appointment date. Subjects were told that they could speak to the endoscopist if they had any questions or anxieties. The remaining subjects with low risk polyps were discharged and offered no further clinical follow up (fig 2). During this phase of the trial only, subjects found to have five or more hyperplastic polyps above the distal rectum were sent for colonoscopy. This group was included since it has been suggested that the presence of metaplastic polyps is a predictor for both synchronous and metachronous adenomas. $^{45}$

ADVERSE PHYSICAL OR PSYCHOLOGICAL EFFECTS The occurrence of adverse physical and psychological effects associated with the entire screening procedure was carefully monitored. Anxiety status was ascertained before and three months after screening using a postal questionnaire (results to be reported later). Subjects were also asked to complete a questionnaire asking about adverse effects on the morning after screening.

\section{STATISTICAL ANALYSIS}

Armitage's test for trend was used to test for differences between the centres and between men and women in the number, size, histology (tubular, tubulovillous, villous) and grade of dysplasia (mild, moderate, severe) of polyps detected at screening FS, comparing for each individual the largest and most advanced polyp detected. The trend test was used for similar comparisons of the pain experienced (none, mild, moderate, severe), of pain experienced versus expected (less painful, the same, more 
Follow up after flexible sigmoidoscopy

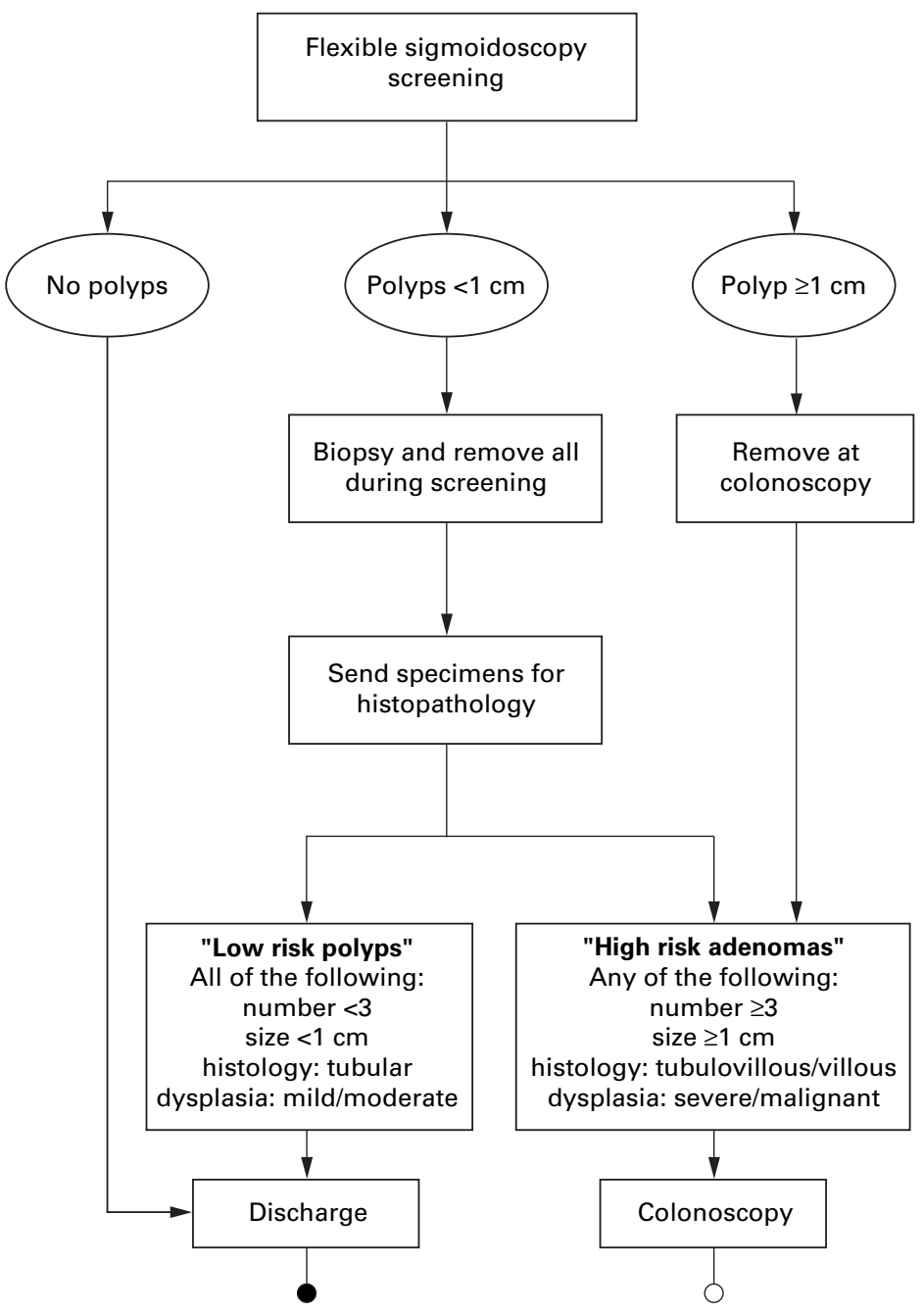

- Subject followed passively through NHS Central Register of the Office of Populations Censuses and Surveys

Colonoscopic surveillance as appropriate

Figure 2 Follow up after flexible sigmoidoscopy.

painful), and of itemised adverse effects (rated none, mild, moderate, severe). All $\mathrm{p}$ values are two sided.

\section{Results}

RESPONSE TO THE "INTEREST IN SCREENING" QUESTIONNAIRE

The initial mailing of the "interest in screening" questionnaire, sent to 3540 subjects in WGC, produced a response rate of $53 \%$; the reminder sent to the 1657 non-responders produced a response rate of $48 \%$, increasing the total response rate to $76 \%$. In total, 2100 $(59 \%)$ responded positively (indicating that they would probably or definitely have the test if invited) and were entered into the trial (table 1).

In LE, the questionnaire plus a reminder was sent to 19706 people, producing a response
Table 1 Response to "interest in screening" questionnaires in Welwyn Garden City and Leicester

\begin{tabular}{lcc}
\hline & Welwyn Garden City & Leicester \\
\hline Definitely yes & $1400(39)$ & $8785(45)$ \\
Probably yes & $700(20)$ & $3253(16)$ \\
Probably no & $260(7)$ & $1724(9)$ \\
Definitely no & $167(5)$ & $1487(7)$ \\
Not recorded & $169(5)$ & $104(1)$ \\
No response & $844(24)$ & $4353(22)$ \\
Total & 3540 & 19706 \\
\hline
\end{tabular}

Values are expressed as number (\%).

rate of $78 \%$. A total of 12199 (61\%) responded positively, and were entered into the trial.

RESPONSE TO INVITATION FOR SCREENING

By the end of the study in WGC, 536 (74\%) of the 719 people invited had attended. Of these, $488(68 \%)$ responded to the initial invitation and the remaining 48 after either a written or telephone reminder. In LE, 1030 individuals were offered an appointment before the end of 1995 and 770 (75\%) had attended by May 1996. Of these, $653(63 \%)$ attended after an initial invitation and 116 after a written reminder ( $51 \%$ of those sent a reminder).

The attendance rate among those who had indicated on the initial questionnaire that they would definitely have the test if invited was $82 \%$ in both centres, compared with $63 \%$ and $47 \%$ respectively in WGC and LE among those who said they would probably attend.

YIELD OF POLYPS AND CANCERS AT FLEXIBLE SIGMOIDOSCOPY

Polyps were removed from slightly more subjects in LE than in WGC (24\% versus $21 \%$ ) due to the removal of significantly more polyps of size $1-5 \mathrm{~mm}$, the majority of which were found to be metaplastic (table 2). Polyps of this size, if they had the typical appearance of metaplastic polyps, were not removed in WGC.

Table 2 Prevalence and characteristics of polyps detected during flexible sigmoidoscopy screeing in Welwyn Garden City and Leicester

\begin{tabular}{lcc}
\hline & $\begin{array}{c}\text { Welwyn Garden City } \\
(n=536)\end{array}$ & $\begin{array}{c}\text { Leicester } \\
(n=749)\end{array}$ \\
\hline Cancer & $4(0.8)$ & $4(0.5)$ \\
Polyps & $108(21)$ & $180(24)$ \\
Total number per person & & \\
1 & $74(14)$ & $118(16)$ \\
2 & $15(3)$ & $33(4)$ \\
$3-4$ & $16(3)$ & $18(2)$ \\
$5-9$ & $3(1)$ & $11(1)$ \\
Size (mm) & & \\
$1-5$ & $67(12)$ & $153(20)$ \\
$6-9$ & $16(3)$ & $11(1)$ \\
$10-14$ & $9(2)$ & $5(1)$ \\
$15+$ & $10(2)$ & $128(1)$ \\
Metaplastic polyps & $68(13)$ & $67(9)$ \\
Adenomas & $53(10)$ & \\
Histology & & $53(7)$ \\
Tubular & $30(6)$ & $10(1)$ \\
Tubulovillous & $19(3)$ & $4(0.5)$ \\
$\quad$ Villous & $4(1)$ & \\
Dysplasia & & $40(5)$ \\
Mild & $17(3)$ & $20(2)$ \\
Moderate & $22(4)$ & $6(1)$ \\
Severe & $13(2)$ & $44(6)$ \\
High risk† & $32(6)$ & \\
\hline
\end{tabular}

Values are expressed as number of subjects (\%).

*Largest, most advanced histopathology.

tAny of the following: $\geqslant 3$ adenomas, $\geqslant 1 \mathrm{~cm}$, tubulovillous, villous, severely dysplastic, malignant, $\geqslant 5$ metaplastic polyps. 
Table 3 Cancers detected during screening

\begin{tabular}{|c|c|c|c|c|c|c|}
\hline & Sex & Age (y) & Site & $\begin{array}{l}\text { Size } \\
(\mathrm{mm})\end{array}$ & $\begin{array}{l}\text { Dukes's } \\
\text { stage }\end{array}$ & Removal \\
\hline \multicolumn{7}{|c|}{ Welwyn Garden City } \\
\hline 1 & M & 57 & $\mathrm{SC}(25 \mathrm{~cm})$ & 9 & A & Sigmoid colectomy \\
\hline 2 & $\mathrm{~F}$ & 63 & $\mathrm{SC}(25 \mathrm{~cm})$ & 20 & $\mathrm{C}$ & Anterior resection \\
\hline 3 & $\mathrm{M}$ & 64 & $\mathrm{SC}(25 \mathrm{~cm})$ & 25 & A & Endoscopic \\
\hline 4 & $\mathrm{~F}$ & 64 & $\mathrm{SC}(20 \mathrm{~cm})$ & 20 & B & Anterior resection \\
\hline \multicolumn{7}{|c|}{ Leicester } \\
\hline 1 & $\mathrm{M}$ & 64 & $\mathrm{RM}(10 \mathrm{~cm})$ & 80 & C & Anterior resection \\
\hline 2 & $\mathrm{~F}$ & 58 & DC $(40 \mathrm{~cm})$ & 60 & A & Endoscopic \\
\hline 3 & $\mathrm{M}$ & 62 & $\mathrm{SC}(25 \mathrm{~cm})$ & 20 & A & Anterior resection \\
\hline 4 & $\mathrm{M}$ & 60 & $\mathrm{RM}(10 \mathrm{~cm})$ & 20 & A & Resectoscope \\
\hline $5^{\star}$ & $\mathrm{F}$ & 63 & $\mathrm{SF}(80 \mathrm{~cm})$ & 50 & $\mathrm{C}$ & Left hemicolectomy \\
\hline
\end{tabular}

*Detected at subsequent colonoscopy.

SC, sigmoid colon; RM, rectum; DC, descending colon; SF, splenic flexure

The prevalence of adenomas was similar in WGC and LE (10\% versus $9 \%$ respectively). However, adenomas in WGC were significantly larger, more villous, and more severely dysplastic than in LE.

High risk polyps (including cancers) were detected in $32(6 \%)$ and $44(6 \%)$ subjects in WGC and LE. An additional four were erroneously classified as high risk in WGC. All were referred for colonoscopy. Two people refused colonoscopy in WGC, but both had already had their distal adenomas removed at FS. At the time of writing, two in LE are awaiting colonoscopy, one of whom has emigrated.

FINDINGS AT COLONOSCOPY

In WGC and LE respectively, 34 and 42 individuals underwent colonoscopy, with complete examinations achieved in $29(85 \%)$ and 31 (74\%). In LE two colonoscopies were required in five patients to complete polypectomy and examine the proximal colon. Of the remaining 11 patients in LE with incomplete colonoscopy, seven underwent double contrast barium enema and the other four will be reviewed in one year. Polyps in the proximal colon were detected in five $(15 \%)$ patients in WGC and five $(12 \%)$ in LE. The proximal polyps in two patients in WGC and in all five in LE had advanced pathological features (at least 10 $\mathrm{mm}$, tubulovillous or villous, or severe dysplasia). A sixth person in LE had a cancer detected at the splenic flexure (see below).

CANCERS

Four cancers were detected among the 536 people screened in WGC $(0.8 \%)$ (table 3$)$. All were located in the sigmoid colon. There were two Dukes's stage A, one Dukes's stage B, and one Dukes's stage C. One patient was treated endoscopically and the rest underwent surgery.

Four cancers were detected at screening among the 749 people screened in LE $(0.5 \%)$. There were three Dukes's stage A and one Dukes's stage C. A fifth, Dukes's stage C cancer, was found at colonoscopy. This was in a 63 year old woman in whom a $4 \mathrm{~mm}$ moderately dysplastic tubulovillous adenoma had been detected in the sigmoid colon. She underwent a colonoscopy at which a $5 \mathrm{~cm}$ cancer was detected in the descending colon.

EXPERIENCES OF THE TEST

Around $80 \%$ in both WGC and LE reported that they had experienced either no or mild pain and in almost half the people screened it was less than expected ( $48 \%$ versus $44 \%$ ). A similar proportion in WGC and LE rated the pain they had experienced as moderate or severe $(18 \%$ versus $14 \%, p=0.6)$ or more painful than they had expected (19\% versus $13 \%$, $\mathrm{p}=0.11$ ), although most described feeling discomfort rather than pain (table 4). Women reported significantly more pain than men.

The most common complaint after the procedure was wind, which was reported as being moderate or severe in $50 \%$ in WGC compared with $24 \%$ in LE $(p<0.0001)$ (table 5). The reduced rate in LE was probably due to the use of carbon dioxide for insufflating the bowel, since carbon dioxide is more rapidly absorbed through the bowel wall than air. The next most frequent complaint was of abdominal pain (WGC 19\% versus LE 12\%) followed by anal irritation (WGC 7\% versus LE 10\%). Very few subjects reported feeling faint or dizzy, suffering from nausea, or incontinence. Sleep disturbance was reported significantly more frequently in LE, probably because the screening clinics were held in the afternoons in LE and in the mornings in WGC.

ADVERSE PHYSICAL REACTIONS

Bleeding was spontaneously reported by seven people $(1.3 \%)$ in WGC and $33(4.6 \%)$ in LE. In 14, the bleeding followed polypectomy. One man was admitted following cold biopsy of a suspected cancer and was discharged the next day. Another man suffered a myocardial infarc-

Table 4 Experience of pain during flexible sigmoidoscopy by sex and trial centre

\begin{tabular}{|c|c|c|c|c|c|c|}
\hline \multirow[b]{2}{*}{ Pain experienced } & \multicolumn{3}{|c|}{ Welwyn Garden City } & \multicolumn{3}{|l|}{ Leicester } \\
\hline & Men $(n=151)$ & Women $(n=184)$ & Total $(n=335)$ & $\operatorname{Men}(n=360)$ & Women $(n=379)$ & Total $(n=739)$ \\
\hline None & $57(38)$ & $40(22)$ & $97(29)$ & $129(37)$ & $94(25)$ & $223(30)$ \\
\hline Mild & $72(48)$ & $101(55)$ & $173(52)$ & $192(33)$ & $218(58)$ & $410(55)$ \\
\hline Moderate & $19(13)$ & $39(21)$ & $58(17)$ & $35(10)$ & $59(16)$ & $94(13)$ \\
\hline Severe & $2(1)$ & $3(2)$ & $5(1)$ & $2(1)$ & $8(2)$ & $10(1)$ \\
\hline Not recorded & $1(1)$ & $\begin{array}{c}1(1) \\
\mathrm{p}(\text { trend })=0.001^{\star}\end{array}$ & $2(1)$ & $2(1)$ & $0(0)$ & $2(1)$ \\
\hline & & & $\mathrm{p}($ trend $)=0.018 \dagger$ & & $\mathrm{p}($ trend $)=0.0001^{\star}$ & \\
\hline Less than expected & $74(49)$ & $88(48)$ & $162(48)$ & $164(46)$ & $162(43)$ & $326(44)$ \\
\hline Same as expected & $45(30)$ & $60(33)$ & $105(31)$ & $151(42)$ & $160(42)$ & $311(42)$ \\
\hline More than expected & $28(18)$ & $35(19)$ & $63(19)$ & $41(11)$ & $54(14)$ & $95(13)$ \\
\hline Not recorded & $4(3)$ & $\begin{array}{l}1(1) \\
p(\text { trend })=0.08^{\star}\end{array}$ & $\begin{array}{c}5(1) \\
p(\text { trend })=0.07 t\end{array}$ & $4(1)$ & $\begin{array}{c}3(1) \\
\mathrm{p}(\text { trend })<0.25^{\star}\end{array}$ & $7(1)$ \\
\hline
\end{tabular}

Values are expressed as number $(\%)$

${ }^{\star} \mathrm{p}$ (trend) for differences between men and women in each centre in ratings, excluding those patients who did not record a response.

$\mathrm{tp}$ (trend) for differences between Welwyn Garden City and Leicester in ratings as above. 
Table 5 Number (\%) of subjects rating itemised adverse effects during or after flexible sigmoidoscopy as moderate or severe as recorded on the morning after the test

\begin{tabular}{lccc}
\hline Adverse effect & $\begin{array}{l}\text { Welwyn Garden City } \\
(n=335)\end{array}$ & $\begin{array}{l}\text { Leicester } \\
(n=734)\end{array}$ & $p$ (trend)* \\
\hline Abdominal pain & $63(19)$ & $87(12)$ & $<0.001$ \\
Nausea/vomiting & $3(1)$ & $7(1)$ & 0.7 \\
Faint/dizzy & $9(3)$ & $15(2)$ & 0.6 \\
Wind & $166(50)$ & $181(24)$ & $<0.001$ \\
Anal irritation & $22(7)$ & $75(10)$ & 0.003 \\
Incontinence & $14(4)$ & $37(5)$ & 0.9 \\
Sleep disturbance & $7(2)$ & $55(7)$ & 0.0002 \\
\hline
\end{tabular}

^For differences between centres in rating of adverse effects from none, mild, moderate to severe.

tion shortly after the test. One woman in WGC suffered a vasovagal attack and another at LE had continuous diarrhoea for several days after taking Picolax. One patient in LE died after developing peritonitis and septicaemia following an open colotomy for removal of a $1 \mathrm{~cm}$ severely dysplastic tubulovillous adenoma at the splenic flexure. Endoscopic polypectomy had been attempted on two occasions but was unsuccessful because of the difficulties in fixing the polyp in a suitable position for snaring.

\section{Discussion}

Two studies have now been completed examining our estimates of rates of interest in and attendance for screening, yield of neoplasia, and adverse effects with a single flexible sigmoidoscopy screen at age 55-64 years. The similarity in the results between the two centres, despite differences in geographical location and socioeconomic status, is evidence of their robustness.

High compliance rates are required in randomised trials, analysed by intention to treat, firstly to maximise the power for a given sample size and secondly to obtain a more accurate estimate of the screening effect. On the assumption that people who attend for screening have similar risks to those who do not, a study with a $50 \%$ compliance rate, when analysed by intention to treat, will observe only $50 \%$ of the true effect of the intervention and will require four times the sample size to achieve the same power. If incidence rates in the non-compliers are higher than in the compliers, an even smaller effect will be observed (this does not appear to be the case in this study, as discussed below). Attendance rates of $74 \%$ were achieved in both centres by sending a preliminary questionnaire designed to identify people who were more likely to attend. The trial participants selected in this way constituted $60 \%$ of those sent the questionnaire. Assuming zero compliance in the rest, the $74 \%$ attendance in the $60 \%$ who indicated that they would attend represents an overall compliance rate of $44 \%$ ( $74 \%$ of $60 \%)$. This figure is similar to the $47 \%$ attendance rate achieved in the feasibility study undertaken in a neighbouring population in Welwyn village. ${ }^{6}$ Thus it appears that the two stage recruitment procedure increased the uptake rate among trial participants by almost $30 \%$.

Based on the results of previous published surveys $^{7-9}$ we had predicted that $8-10 \%$ of screened subjects would have adenomas and that $3-5 \%$ would have high risk adenomas and require colonoscopy. Using population incidence rates and assuming a lead time for the diagnosis of symptomatic cancer of three years, we had anticipated that the cancer detection rate would be 1.5 per 1000 . The rates of detection in WGC and LE (7 per 1000 in both centres: $95 \%$ confidence intervals 2 to 19 and 2 to 16 per 1000 respectively) were significantly higher than expected. There are three explanations for this. Firstly it is possible that the lead time for cancers detected at endoscopy is longer than has been suggested for cancers detected using faecal occult blood tests (FOBT). However the stage distribution of the cancers in this study was similar to that detected in the prevalence round of screening with FOBT in the randomised trial in Nottingham ${ }^{1}$ (50\% Dukes's A cases in each series), which is evidence against this hypothesis. A third explanation is that our questionnaire selects people who are at increased risk of cancer. That the detection rate of high risk polyps ( $6 \%$ in both centres) was slightly higher than predicted suggests this may have been a higher risk group. In an associated study of the predictors of interest in screening in this trial we found that those who express an interest (and are entered into the trial) report more colorectal symptoms than those who are not interested (Wardle, personal communication).

Unlike most other screening protocols this regimen attempts to ensure that the majority of screened subjects need attend only once. All small polyps are therefore removed at screening unless there is a medical contraindication. Colonoscopy is reserved for those found to have high risk polyps which include any of the following: three or more adenomas, tubulovillous or villous histology, or severe dysplasia. Those with only one or two small (not greater than $1 \mathrm{~cm}$ ), tubular, mildly dysplastic adenomas are discharged and undergo no further clinical follow up. This is contrary to the established but unsustainable practice of unselective referral for colonoscopy for all patients harbouring adenomas. ${ }^{10}$ However, there is much evidence that small tubular distal adenomas are associated with an increased risk of small low risk adenomas, but not of advanced neoplasia or cancer, in the proximal colon. ${ }^{11-13}$

It was reassuring that more than $80 \%$ of people experienced only mild discomfort or none at all, and that it was rated as severe by only $1.5 \%$. Bleeding occurred in a total of 40 subjects in the two centres but was generally mild and short lived; it followed polypectomy in about one third (14 subjects) and was associated with complaints of prolonged diarrhoea in most of the other subjects.

The findings in these studies show the likely level of uptake, yield of neoplasia, and side effects generated by implementation of the main trial protocol. Accordingly the trial has been started in 12 others centres across the United Kingdom; it is anticipated that data on cancer incidence will become available in 10 years. 


\section{Conclusion}

These pilot studies have shown that the protocol for the multicentre randomised trial of once only flexible sigmoidoscopy is logistically feasible and is being adopted in the main trial. Compliance rates are high, the yield of neoplasia is as expected, and the examination appears to be acceptable to screened subjects. An unexpected result was the high rate of detection of cancers. There were no serious adverse effects of sigmoidoscopy or colonoscopy. The death of a patient following surgery emphasises the necessity for a randomised trial, not only to quantify the benefits but also to document adverse effects, before widespread implementation of screening.

The studies were funded by Imperial Cancer Research Fund (ICRF), the Medical Research Council (MRC), NHS R\&D, and KeyMed Ltd. We thank all the general practitioners and patients who participated and Dr J F Mayberry, Ms S Daniels, Ms A Read, Dr A C B Wicks, Dr H Mackay, Dr Y Rees, Mr M J Kelly, Sisters J Lawson and S Burgin, and the endoscopy and day care nurses at Leicester General Hospital; Mrs Jennife Pankhurst, Sister Tracey Lewis, Sister Kay Allwright, Sister Dawn Webb, Mrs Pam Rogers, and the endoscopy nursing staf of the Queen's Wing, QEII Hospital, Welwyn Garden City; D Lin Squires, Mr Steve Edmeades (Bioinformatics Unit), Mrs J Hickman, Dr P Sasieni (Mathematics, Statistics and Epidemiology Unit), ICRF; Ms Clare Baron, Ms Sheri Edwards, Dr C B Williams, Professor I Talbot, Ms Terri Lichay, and Mrs S Gardiner at St Mark's Hospital.
1 Hardcastle J, Chamberlain J, Robinson M, et al. Randomised controlled trial of faecal-occult-blood screening domised controlled trial of faecal-occult-blood

2 Kronborg O, Fenger C, Olsen J, et al. Randomised study of screening for colorectal cancer with faecal-occult-blood test. Lancet 1996;348:1467-71.

3 Atkin WS, Cuzick J, Northover JMA, et al. Prevention of colorectal cancer by once-only sigmoidoscopy. Lancet 1993;341:736-40.

4 Kellokumpu I, Kyllonen L. Multiple adenomas and synchronous hyperplastic polyps as predictors of metachronous colorectal adenomas. Ann Chir Gynaecol 1991;80: 30-5.

5 Jass J, Young P, Robinson E. Predictors of presence, multiplicity, size and dysplasia of colorectal adenomas. A necropsy study in New Zealand. Gut 1992;33:1508-14.

6 Verne J. Screening for colorectal neoplasia in primary care. $\mathrm{PhD}$ thesis. London, 1993

7 McCallum RW, Meyer CT, Marignani P, et al. Flexible sigmoidoscopy: diagnostic yield in 1015 patients. Am $\mathcal{F}$ Gastroenterol 1984;79:433-7.

8 Rosevelt J, Frankl H. Colorectal cancer screening by nurse practitioner using $60-\mathrm{cm}$ flexible fiberoptic sigmoidoscope. Dig Dis Sci 1984;29:161-3.

9 Shida H, Yamamoto T. Fiberoptic sigmoidoscopy as the first screening procedure for colorectal neoplasms in a symptomatic population. Dis Colon Rectum 1989;32:404-8.

10 Woodman C, Prior P, Joseph RAW. Prospects for the secondary prevention of colorectal cancer by screening by
flexible sigmoidoscopy. $\mathcal{M}$ Med Screen $1995 ; 2: 71-8$.

11 Grossman S, Milos ML, Tekawa IS, et al. Colonoscopic screening of persons with suspected risk factors for colon cancer: II. Past history of colorectal neoplasms. Gastroenterology 1989;96:299-306.

2 Gatteschi B, Costantini M, Bruzzi P, et al. Univariate and multivariate analyses of the relationship between adenocarcinoma and solitary and multiple adenomas in colorectal adenoma patients. Int $\mathcal{F}$ Cancer 1991;49:509-12.

13 Zarchy TM, Ershoff D. Do characteristics of adenomas on flexible sigmoidoscopy predict advanced lesions on baseline colonoscopy? Gastroenterology 1994;106:1501-4. 